\title{
G-Quadruplex Assembly by Ribosomal DNA: Emerging Roles in Disease Pathogenesis and Cancer Biology
}

\author{
Arindam Datta $^{a}$ Kevin J. Pollock ${ }^{b}$ Karen A. Kormuth ${ }^{b}$ Robert M. Brosh Jr ${ }^{a}$

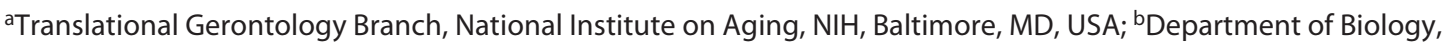 \\ Bethany College, Bethany, WV, USA
}

\section{Keywords}

Ribosomal DNA · G-quadruplex · Helicase · Aging · Cancer · DNA damage $\cdot$ Genomic instability $\cdot$ Transcription

\begin{abstract}
Unique repetitive elements of the eukaryotic genome can be problematic for cellular DNA replication and transcription and pose a source of genomic instability. Human ribosomal DNA (rDNA) exists as repeating units clustered together on several chromosomes. Understanding the molecular mechanisms whereby rDNA interferes with normal genome homeostasis is the subject of this review. We discuss the instability of rDNA as a driver of senescence and the important roles of helicases to suppress its deleterious effects. The propensity of rDNA that is rich in guanine bases to form G-quadruplexes (G4) is discussed and evaluated in disease pathogenesis. Targeting G4 in the ribosomes and other chromosomal loci may represent a useful synthetic lethal approach to combating cancer.
\end{abstract}

(c) 2021 This is a work of the U.S. Government and is not subject to copyright protection in the United States. Foreign copyrights may apply. Published by S. Karger AG, Basel

\section{Introduction}

Ribosomal DNA (rDNA) refers to the genes which are transcribed, processed, folded, and assembled with ribosomal proteins to become the functional core of the ribosome. Eukaryotic rDNA is uniquely structured as a series of repeating units that template the $5 \mathrm{~S}$ rRNA (transcribed by RNA pol III) and a polycistronic unit for the remaining rRNAs and transcribed spacer elements (transcribed by RNA pol I) (Fig. 1). rDNA transcription must be tightly regulated to maintain genome stability and appropriate protein synthesis and cell growth rates [Kief and Warner, 1981; Ide et al., 2010]. Eukaryotic rDNA has also been predicted to have a high propensity for alternate secondary structure formation, specifically guanine-quadruplexes (G4) [Hanakahi et al., 1999; Hershman et al., 2008]. G4 that form within these genes may have a significant impact on the regulation of transcription and genome stability, with important implications for processes associated with dysregulated cell growth, like aging and cancer.

G4 are nucleic acid secondary structures resulting from folding of G-rich motifs (Fig. 2). G4 are stabilized by Hoogsteen hydrogen bond pairing within planar Gtetrads that stack on top of one another. The scope of genomic G4 biology, which can behave as cis-acting regula-

Correspondence to:

Karen A. Kormuth, kkormuth@bethanywv.edu

Robert M. Brosh Jr, broshr@mail.nih.gov 


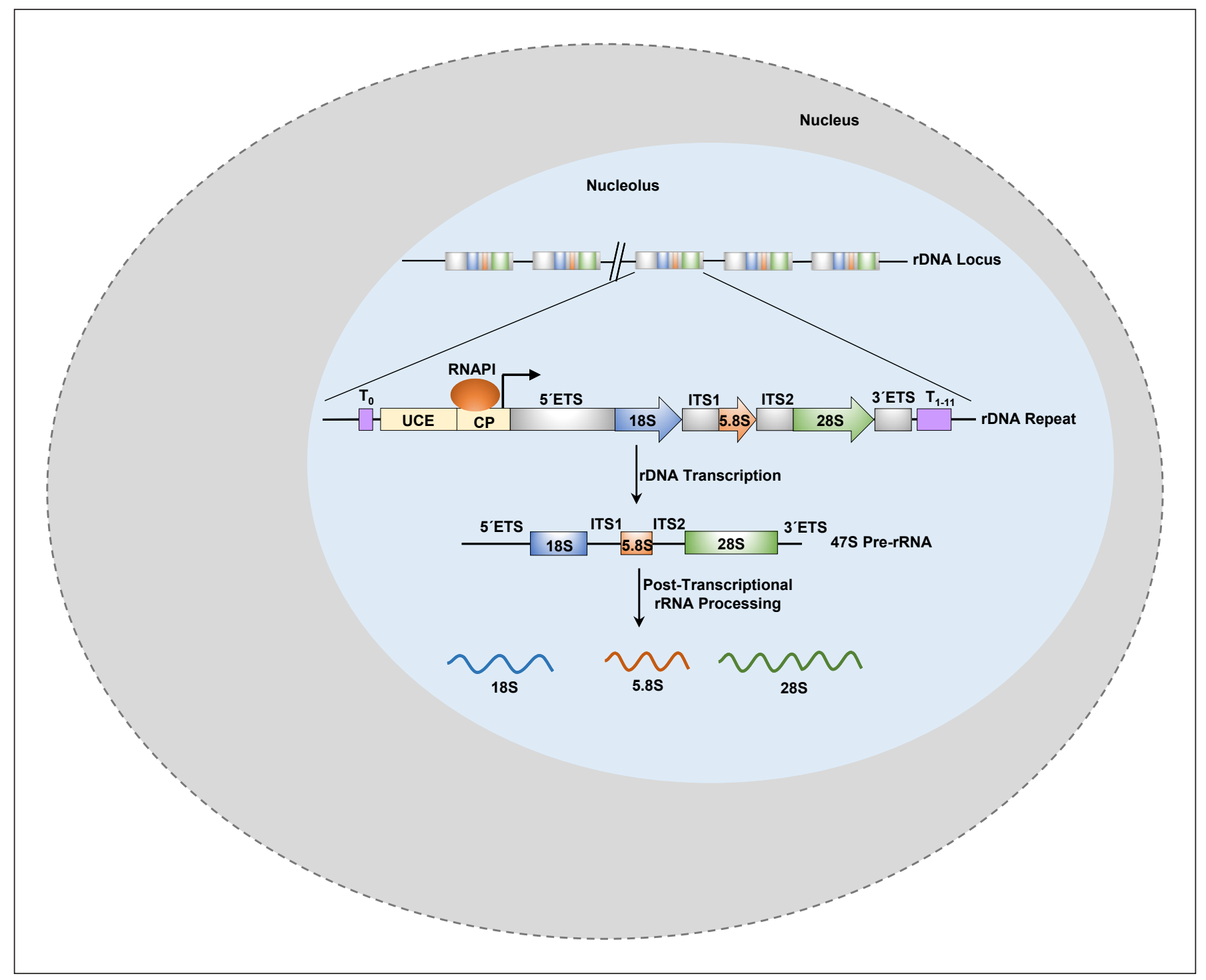

Fig. 1. Transcription of ribosomal DNA (rDNA) in human cells. The synthesis of ribosomal RNAs (18S, 5.8S, and 28S) occurs inside the nucleolus in human cells. The human rDNA locus is organized as multiple repeating rDNA units each approximately 43 $\mathrm{kb}$ long. Each rDNA repeat consists of an rRNA gene, promoter (core promoter, $\mathrm{CP}$; and upstream control element, UCE), regulatory elements, and upstream $\left(\mathrm{T}_{0}\right)$ and downstream $\left(\mathrm{T}_{1-11}\right)$ terminator sequences [Goodfellow and Zomerdijk, 2013; Srivastava et al., 2016].rRNA genes are flanked by an approximately 30-kb-long

tory elements in a variety of cellular processes, is continuing to be uncovered. The most recent evidence-based estimates of $>700,000 \mathrm{G} 4$ loci in the human genome [Chambers et al., 2015], are over 1.5 times greater than earlier computational estimates [Huppert and Balasubramanian, 2005; Eddy and Maizels, 2006; Kikin et al., 2006]. This number was validated through the development of a intergenic spacer that contains the promoter and regulatory sequences. Along with the sequences for $18 \mathrm{~S}, 5.8 \mathrm{~S}$, and $28 \mathrm{~S}$ rRNAs, the coding region of an rRNA gene contains 2 internal transcribed spacer (ITS) regions, and $5^{\prime}$ and $3^{\prime}$ external transcribed spacer (ETS) sequences [Peculis, 2002; McStay and Grummt, 2008; Goodfellow and Zomerdijk, 2013]. Ribosomal RNAs are transcribed by RNA pol I as a polycistronic precursor transcript (47S pre-rRNA) which undergoes further processing and posttranscriptional modifications to generate $18 \mathrm{~S}, 5.8 \mathrm{~S}$, and $28 \mathrm{~S}$ rRNA molecules.

high-throughput, genome-wide method using sequencing-based approaches to detect $\mathrm{G} 4$ formation induced by a G4-stabilizing small molecule [Chambers et al., 2015]. Another landmark study published in 2013 achieved visualization of nuclear G4 in cultured human cancer cells using a G4-specific antibody, BG4 [Biffi et al., 2013]. A caveat of these reports is that introduction of an antibody 
Fig. 2. Schematics of G-quadruplex (G4) structural topology. a Typical G-rich quadruplex-forming motif, having 4 tracts of 2-4 Gs each, separated by spacer sequences. In the folded structure, the Gs interact via Hoogsteen $\mathrm{H}$-bonding to form planar tetrads that stack vertically, with all 3 loop sequences located outside of the tetrad core. b Intermolecular quadruplex formed from the interaction of $2 \mathrm{G}$-rich nucleic acid strands. In this cartoon, 2 G-tracts are derived from the light blue strand, and the remaining 2 are from the dark blue strand, forming a hybrid structure. For simplicity, only parallel G-quadruplexes (having all 4 G-tracts with uniform polarity) are shown in this figure.

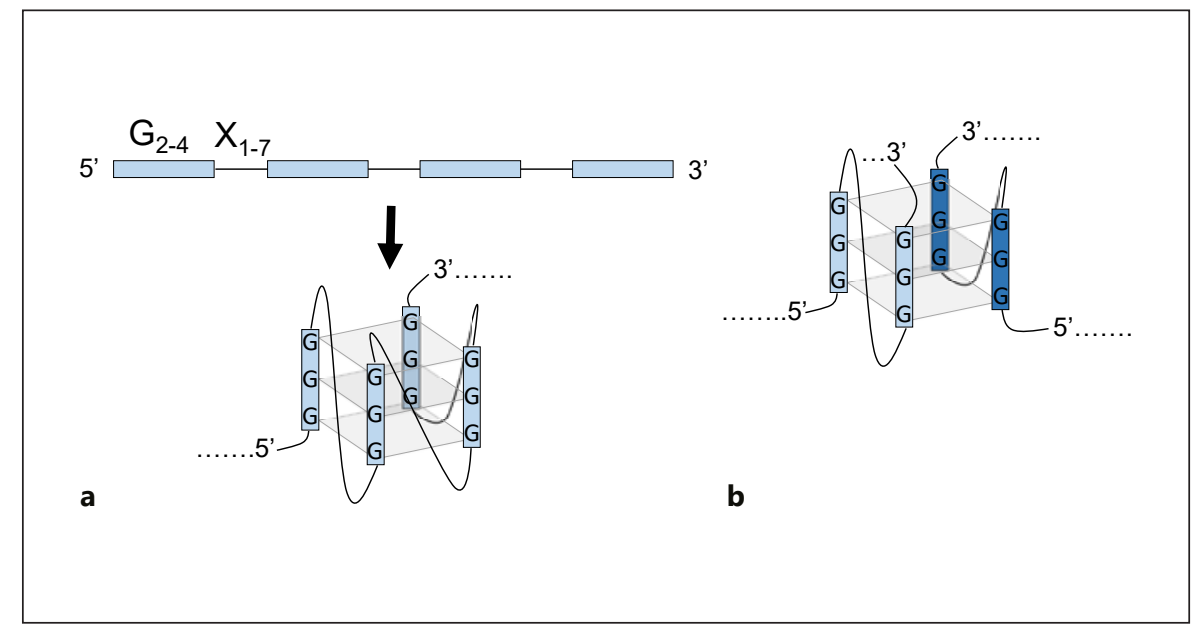

or G4-stabilizing molecule may induce some artifactual formation of these structures. However, the overarching conclusion is that G4-forming motifs are prevalent throughout the human genome and are likely to have farreaching regulatory and functional consequences during DNA replication and gene expression. While whole-genome analyses have paved the way forward for G4 biology, continued investigation of G4 located in specific functional regions is needed to define their unique roles inside the cell. Among these, ribosomal genes are among the most G4-rich loci in the eukaryotic genome [Hanakahi et al., 1999; Hershman et al., 2008].

In this review, we will provide an overview of methods for identification and characterization of genomic G4 localized to ribosomal genes (rDNA). Evidence for G4 regulation of rDNA gene expression and genome stability is discussed, with a particular focus on G4-resolving helicases in cell senescence and cancer. Modern synthetic lethal approaches to anti-cancer therapies have involved G4-targeting ligands, and future identification of the mechanisms by which G4 regulate rDNA gene function will be important to move these approaches forward.

\section{Conventional G4 Motifs and Prediction of G4 Motifs}

The most frequently recognized intramolecular $\mathrm{G} 4$ motif is $G_{m}-X_{n}-G_{m}-X_{o}-G_{m}-X_{p}-G_{m}$, where each G-tract $\left(G_{m}\right)$ is separated by loops $\left(X_{n}, X_{o}\right.$, and $\left.X_{p}\right)$, and $n, o$, and $\mathrm{p}$ imply there can be many different combinations of nucleotides, including $G$, of various lengths up to $\sim 7$ nucleotides [Burge et al., 2006]. Following this canonical motif, putative $\mathrm{G} 4$ have been identified in various functional regions of the eukaryotic genome by computational predic-

Ribosomal DNA, G-Quadruplexes, and Disease tion software packages, including Quadparser [Huppert and Balasubramanian, 2005], Quadruplex Forming Grich Sequences (QGRS) Mapper [Kikin et al., 2006], and G-4 pred [Hershman et al., 2008]. More recently, unconventional G4 motifs have been identified, including those with long loops [Kormuth et al., 2016], bulging loops [Mukundan and Phan, 2013], and multiplex hairpin/G4 topology [Palumbo et al., 2009]. Interrogation of noncanonical G4 motifs is an ongoing area of research.

\section{Biochemical Characterization of G4}

Standard biochemical analysis protocols for putative G4 include spectroscopic approaches to identify in vitro G4 folding topology and structural stability. Initially, putative G4 should be assessed for in vitro folding and thermal stability to determine whether they are likely to form stable structures at physiological temperatures. G4 folding is also supported by the presence of monovalent cations, usually $\mathrm{K}^{+}$[Hardin et al., 1992]. Historically, the approach to biochemical analysis of $\mathrm{G} 4$ oligomers has been to compare data using $\mathrm{K}^{+}$- versus $\mathrm{Li}^{+}$-containing buffers, as the ionic radius of $\mathrm{Li}^{+}$is too small to support tetrad stability [Hardin et al., 1992]. Thermal stability can be directly assessed by UV melting spectroscopy, which produces a characteristic hypochromic shift in the absorbance spectrum at $295 \mathrm{~nm}$ and allows for direct measurement of melting temperature from the first derivative of the melting curve. Similarly, thermal difference spectroscopy (TDS) can detect various nucleic acid secondary structures in vitro [Mergny et al., 2005]. TDS data are gathered by subtracting the UV absorbance spectrum of the folded structure from that of the unfolded states, 
which produces a G4-specific inversion at $295 \mathrm{~nm}$. Circular dichroism spectropolarimetry is also routinely used to detect G4 formation and topology [Paramasivan et al., 2007]. G4 having parallel topology, with all 4 G-tracts oriented in the same direction, produce a circular dichroism spectrum with a maximum absorbance near $260 \mathrm{~nm}$ and a minimum at $240 \mathrm{~nm}$, while antiparallel structures are detected by spectra with a maximum near $295 \mathrm{~nm}$ and a minimum near $260 \mathrm{~nm}$ [Del Villar-Guerra et al., 2018]. More complex topologies or heterogeneous populations cannot always be resolved using the method. Regardless of the biological system, in vitro analysis of G4 is routinely used to support in vivo functional analyses.

\section{Evidence for G4 in rDNA and Regulation of Gene Expression}

Within eukaryotic genomes, the ribosomal genes, or rDNA, are among the most enriched for putative quadruplex-forming sequences [Hanakahi et al., 1999; Hershman et al., 2008]. Very little is known about the activity of rDNA G4. rDNA provides the genetic blueprint for rRNA transcription, and subsequent folding, processing, and assembly into the mature ribosome. Tight control of ribosome biosynthesis is required for cells to maintain proper protein synthesis and growth rates [Kief and Warner, 1981].rDNA G4, therefore, could regulate processes, like cancer and aging, related to cell growth and proliferation. rDNA genes exist as a series of repeating gene copy units, which in humans are distributed across 5 chromosomes (47S rDNA), with $5 \mathrm{~S}$ rDNA repeats clustered together on chromosome 1 [Warmerdam and Wolthuis, 2019]. Evolution has driven eukaryotes to retain extra copies of rDNA to maintain genome stability, while only a subset of these copies is expressed at any given time to limit cell toxicity [Ide et al., 2010].

In humans, rDNA is particularly G-rich in both the spacer regions and those that template mature rRNA [Hanakahi et al., 1999]. Most of these G-rich motifs are located in the non-template strand, suggesting that G4 formation has the potential to modulate processes at both the DNA and RNA levels [Hanakahi et al., 1999]. Not all G4 motifs in the rDNA follow the canonical pattern, where G-tracts range in length from 2 to 4 Gs separated by loop sequences up to 7 nucleotides in length [Burge et al., 2006]. Putative quadruplex-forming sequences from the yeast, Saccharomyces cerevisiae, rDNA with especially long loops have been previously characterized, which fold into stable G4 structures in vitro in conditions consistent with the intracellular environment [Kormuth et al., 2016]. This result is consistent with whole-genome G4-seq data, which identified greater than 450,000 G4 that fall outside of the classical G4 motif definition [Chambers et al., 2015]. Together, these results highlight the breadth of possibilities for G4-mediated regulation of rDNA stability and processing.

While much of the focus of quadruplex research has been placed on DNA, G4 are not necessarily restricted to the genome. Transcriptomic G4 may regulate gene expression at the RNA level either co- or post-transcriptionally. While quadruplexes have not been identified in the mature eukaryotic ribosome [Ben-Shem et al., 2010], the presence of putative G4 motifs in the rRNA hints at possible roles in transcription, rRNA processing, and/or ribosome assembly. RNA G4 are naturally more thermally stable than their DNA counterparts in vitro, and experience fewer structural constraints compared to duplex-forming double-stranded DNA [Joachimi et al., 2009; Kwok et al., 2016; Song et al., 2016]. However, the prevalence of stable RNA G4 in vivo may be tightly controlled by interactions with G4 helicases and other trans-acting factors present in the cell [Guo and Bartel, 2016]. However, various studies have suggested that RNA G4 may take on a variety of functions, including modulating mRNA stability [Ibrahim et al., 2018], telomere homeostasis [Millevoi et al., 2012], and translation initiation [Bugaut and Balasubramanian, 2012]. Apart from intramolecular RNA quadruplexes, studies of human mitochondrial DNA have provided evidence for the formation of DNA/RNA intermolecular heteroquadruplexes during transcription elongation [Zhang et al., 2014]. This type of hybrid structure may be particularly important in highly transcribed regions of the genome, as R-loops are melted by additional polymerases elongating along the same template [Zhang et al., 2014]. While a role for RNA or hybrid G4 function in rDNA gene expression has yet to be identified, it is reasonable to consider that similar mechanisms may be involved in finetuning transcription levels and/or maintaining genome stability within the nucleolus.

rDNA G4 likely act through interactions with proteins to regulate transcription. For example, human nucleolin protein, which has high affinity for both duplex and quadruplex DNA, localizes to sites of active rDNA transcription in the nucleolus [Hanakahi et al., 1999]. Another nucleolar protein related to cancer, nucleophosmin, readily hybridizes to rDNA G4 structures, and its proper cellular localization is related to certain malignancies [Chiarella et al., 2013]. Therefore, assessment of interactions between proteins/small molecule ligands and quadruplex 
DNA can provide important insight into to the role of these structures, especially within the rDNA. These interactions can be quantified in vitro using standard electrophoretic mobility shift assays. Additionally, kinetic data can be produced using surface plasmon resonance to measure on and off-rates for ligand binding to a surface immobilized G4 oligomer. The combination of such studies with in vivo approaches will illuminate the biological relevance and druggability of rDNA G4.

\section{Instability of rDNA as a Driver of Accelerated Aging}

The essential nature of rDNA with its vast number of repetitive DNA sequences that potentially form secondary structures and are difficult to replicate faithfully suggests that it represents a particular locus of genomic DNA that is implicated in cellular decline with age and agerelated disease [Storci et al., 2020]. While this hypothesis remains to be fully tested, it has not escaped the attention of gerontologists and DNA biologists in general to designate this concept with the term "replicative ribosomal theory of aging" [Kobayashi, 2008]. Indeed in vitro cell aging and gerontological decline characteristic of aging diseases may very well share in common the instability of rDNA loci that accompanies senescence [Gorgoulis et al., 2019]. In addition to the detrimental effects of DNA damage on cellular function [Petr et al., 2020], an emerging hypothesis is that the generation of cytoplasmic or extracellular DNA, as well as RNA, elicits cellular responses. It is plausible that the instability of certain chromosomal loci (e.g., rDNA) leads to pro-inflammatory activation of senescent cells and promotes widespread inflammaging through mechanisms which remain to be fully characterized. For example, work from the Raney lab suggested that G4 DNA has a propensity to accumulate in the cytoplasm of human cells and promote stress granule assembly enhanced by oxidative stress [Byrd et al., 2016]. While this phenomenon and the driving elements behind nuclear and/or mitochondrial DNA extrusion remain to be substantiated in experimental systems, it is plausible that the propensity of guanine-rich elements to form G4 may be directly implicated.

\section{Evidence for Helicase Involvement in rDNA Stability}

Several pieces of evidence suggest that G4-resolving helicases play important roles in leveling out unusual DNA structures to allow smooth replication and tran-

Ribosomal DNA, G-Quadruplexes, and Disease scription [Bharti et al., 2016; Mendoza et al., 2016; Sauer and Paeschke 2017]; however, strong biological evidence that helicases play a primary role in resolving G4 structures that form within the highly guanine-rich rDNA repeats remains to be definitively shown. The first evidence that a DNA helicase plays a role in promoting fork progression past G4 structures existing in rDNA (as well as other loci) and preserves the stability of the DNA sequence proximal to or encoding the G4 motifs was garnered from studies of the highly conserved fission yeast Pfh1 helicase. Using a whole genome sequencing chromatin immunoprecipitation (ChIP-seq) approach, Sabouri et al. [2014] determined that Pfh 1 was enriched at the rDNA of asynchronously growing cells, but not at the G4 motifs in the rDNA. However, while it was evident that the loss of Pfh 1 caused replication fork pausing and DNA damage near G4 motifs, it was unclear from the analysis if either was significantly increased at the ribosomal G4 motifs. However, in a follow-up study, the Sabouri lab used a more sensitive ChIP-qPCR approach with cells expressing an epitope-tagged Pfh 1 and found enriched binding to rDNA G4 during the S-phase of replicative DNA synthesis [Wallgren et al., 2016]. Purified recombinant Pfh1 helicase protein was observed to preferentially bind rDNA in vitro and unwind model rDNA G4 structures in an intrinsic ATPase-dependent manner [Wallgren et al., 2016]. Although ChIP studies with S. cerevisiae cells expressing epitope-tagged Pif1 were performed in other work, which showed an enrichment of Pif1 binding to nuclear G4 motifs, rDNA was excluded from the study due to the difficulty of analyzing the multi-copy DNA sequence [Paeschke et al., 2011]. Schizosaccharomyces pombe Pfh1 was found to be important for efficient fork progression in $\mathrm{rDNA}$ which was attributed to stable protein binding to replication fork block regions in the rDNA [Sabouri et al., 2012]. Further studies are required to determine if Pif1 family members act upon persistent ribosomal G4 DNA structures to allow smooth fork progression, but it seems probable given the evidence that helicases from this conserved family act upon G4 structures at other chromosomal loci and difficult-to-replicate sequences in vivo [for review, see Sabouri, 2017; Sauer and Paeschke, 2017; Muellner and Schmidt, 2020]. Nonetheless, it remains to be shown that Pif1 plays a significant role to resolve ribosomal G4 DNA in human cells.

Model genetic organisms have proven to be highly useful for studying unusual defects in cellular nucleic acid metabolism and are likely to apply in characterizing the functional roles of DNA helicases in genome homeostasis at specific chromosomal loci such as rDNA, telomeres, or 
centromeres. This issue becomes particularly important when the molecular in vivo functions of gene products defective in hereditary disorders are elusive, as is the case for the chromosomal instability disorders Fanconi anemia and Hoyeraal-Hreidarsson syndrome, resulting from bi-allelic mutations in the sequence-related helicase genes FANCJ [Brosh and Cantor, 2014] and RTEL1 [Vannier et al., 2014], respectively. While it is generally believed that both diseases are defective in genomic DNA maintenance and the replication stress response, their precise roles in chromatin biology remain a conundrum. The Puchta lab, specializing in Arabidopsis, investigated the role of the human homologues FANCJ and RTEL1 in genome stability and DNA repair by characterizing gene knockouts in the Brassicaceae family member related to cabbage and mustard [Dorn et al., 2019]. While a deficiency in the Arabidopsis FANCJ homolog resulted in the expected defect in repair of interstrand cross-links, a new finding was that FANCJ, and its sequence-related Fe-S cluster helicase RTEL1, operate in parallel pathways to maintain rDNA stability, as evidenced by the disruption of $45 \mathrm{~S}$ rDNA repeats. Importantly, both FANCJ and RTEL1 of Arabidopsis are independently required for replicationassociated DNA repair in the root meristem, indicative of a bona fide in vivo function. While the authors postulated that the $2 \mathrm{Fe}-\mathrm{S}$ helicases may help to preserve rDNA by unwinding G4 structures that are highly abundant at the locus, no direct experimental evidence was presented to defend this working hypothesis. Nonetheless, the findings from this work provide the first strong biological evidence that a molecular defect in rDNA management may be a fundamental problem in eukaryotic cells that are deficient in FANCJ or RTEL1.

Dna2 is an evolutionarily conserved eukaryotic helicase-nuclease implicated in cellular DNA replication by its involvement in the maturation of Okazaki fragments and processing of regressed replication forks arising from DNA synthesis stalling [Zheng L et al., 2020]. Recently, a role of yeast Dna2 to suppress insertions of large DNA tracts $(0.01-1.5 \mathrm{~kb})$, such as those that originate from rDNA, at sites of double-strand breaks was evidenced [Yu et al., 2018]. The insertions were not lost from their original loci, indicating that they had been duplicated. They were preferentially localized in fragile regions of chromosomes (e.g., replication origins, R-loops, telomeres, centromeres, etc.) and were dependent on nonhomologous end-joining. It was suggested that the size of the insertions was comparable to those reported in human cancers [Li et al., 2020], prompting further studies in their origin and mechanism of formation.
RECQL5, one of the 5 human RecQ helicases [Hamadeh and Lansdorp, 2020] has been implicated to counteract replication fork stalling of genes transcribed by RNA pol I as well as RNA pol II [Urban et al., 2016]. Work from the Janscak lab and others support a seminal role of RECQL5 to resolve collisions of cellular DNA replication and transcription [Andrs et al., 2020], suggesting a testable model for how the helicase enables RNA pol I to transcribe $\mathrm{rDNA}$ in an effective manner. Indeed, a model has been proposed for plausible roles of RECQL5 to manage collisions between the replication and RNA pol II transcription machineries [Andrs et al., 2020], but whether this applies to RNA pol I and what deviations exist remains to be further studied. Although evidence is lacking that RECQL5 directly resolves G4 structures at the rDNA repeats, the helicase has been reported to destabilize G4 DNA substrates in vitro, albeit at a reduced capacity compared to the RECQ helicases WRN or BLM mutated in Werner syndrome and Bloom syndrome, respectively [Budhathoki et al., 2016].

Although details remain to be determined, it is probable that DNA helicases which resolve G4 structures (or other unusual nucleic acid structures) that form in rDNA play a role in their smooth replication. Figure 3 depicts DNA helicases of interest that may have direct roles in rDNA metabolism. Both WRN and BLM helicases bind tightly to oligonucleotide-based G4 DNA structures in vitro and resolve them [Mohaghegh et al., 2001; Kamath-Loeb et al., 2012; Chatterjee et al., 2014], suggesting they play prominent roles in the metabolism of G4 structures that form in nuclear G4 DNA and possibly G4 rDNA. Indeed, WRN (and BLM [Gao et al., 2003]) is enriched in nucleoli (where rDNA resides) in a manner dependent on the cell cycle and DNA damage/replication stress [Gray et al., 1998], suggesting a specialized role in rDNA G4 transactions. In cycling cells, WRN is enriched in transcriptionally active nucleoli but relocalizes to nucleoplasmic foci when cells are made quiescent or exposed to the DNA damaging agent 4-nitroquinoline-1-oxide [Gray et al., 1998]. Experimental evidence demonstrated that WRN resides in an RNA pol I complex and found to increase rRNA transcription [Shiratori et al., 2002], but G4 was not directly implicated in this process.

The major nucleolar protein, nucleolin, binds tightly to G4 DNA and inhibits its resolution by WRN, which may serve a regulatory function [Indig et al., 2012]. In human cells, deficiency of WRN or BLM results in elevated staining of G4, preferentially seen at telomeres [Drosopoulos et al., 2015], providing further evidence that the 


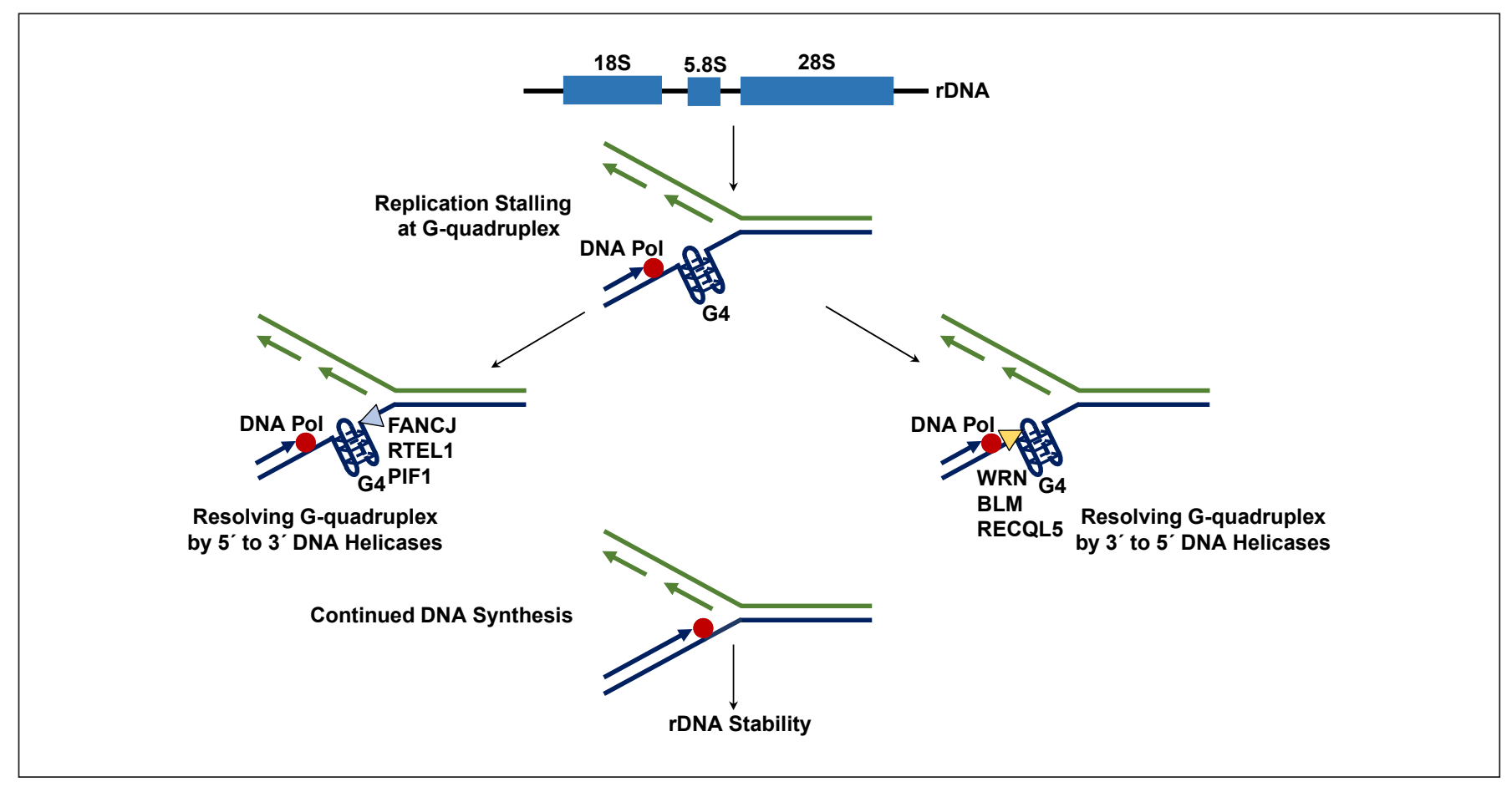

Fig. 3. Potential involvement of G-quadruplex (G4)-resolving helicases in rDNA stability maintenance. G4-resolving helicases help preserve rDNA stability by facilitating replication of $\mathrm{rDNA}$ repeat regions prone to form $\mathrm{G} 4$ structures. $5^{\prime}-3^{\prime}$ helicases such as FANCJ, RTEL1, and PIF1 or $3^{\prime}-5^{\prime}$ helicases such as WRN, BLM, and RECQL5 resolve G4 structures formed ahead of the replicative

RecQ helicases facilitate telomeric DNA synthesis and stability in G4-forming chromosomal regions. It is conceivable that WRN and BLM help to suppress recombination of repetitive G4-forming DNA sequences. Interestingly, a deficiency of the RecQ helicase Sgs1 in S. cerevisiae causes nucleolar fragmentation and deletion of rDNA circles, a phenomenon that plays a role in aging for yeast [Sinclair and Guarente, 1997; Sinclair et al., 1997]. However, it is yet unclear if rDNA circle formation is important for human aging.

In addition to DNA helicases, other cellular enzymes may play a role in G4 DNA metabolism. The Maizels lab discovered a human nuclease specific for G4 DNA [Sun et al., 2001]. Although a role in rDNA stability or function was not examined in that work, it was noted that the G4 nucleolytic activity was elevated in B cells, suggesting a potential role in immunoglobulin switch recombination which occurs at G-rich sequences. Other G4-specific proteins (e.g., nucleolin, discussed below) are thought to have important roles in the metabolism of G4 which may bear upon rDNA.

Ribosomal DNA, G-Quadruplexes, and Disease polymerase and facilitate smooth DNA synthesis. Note that while helicases acting on the leading strand template are depicted, functional roles of helicases acting on the lagging strand template in rDNA are also plausible. For simplicity, intermolecular G4 structures formed by multiple strands (DNA and/or RNA) are not shown.

\section{Plausible Involvement of G4 in Disease Pathogenesis Arising from Defective rDNA Metabolism}

The hereditary DNA repair and transcription disorder Cockayne syndrome (CS) has long been known to have defective nucleic acid metabolism, contributing to the premature aging and congenital abnormalities of the disease [Karikkineth et al., 2017]. However, despite the many labs who have investigated its molecular pathogenesis, CS remains an enigma. Several studies suggested that cells from CS patients are characterized by a deficiency in transcription mediated by RNA pol I [Christians and Hanawalt, 1994; Bradsher et al., 2002; Yuan et al., 2007; Lebedev et al., 2008; Koch et al., 2014], as well as RNA pol II and repair coupled to RNA pol II stalling [Henning et al., 1995; Balajee et al., 1997; Dianov et al., 1997; Selby and Sancar, 1997; Tantin et al., 1997; van Gool et al., 1997; Tantin, 1998; Iyer et al., 2004; Sarker et al., 2005; Fousteri et al., 2006]. Scheibye-Knudsen et al. [2016] found that defective rDNA transcription due to loss of either CS complementation group gene (CSA, CSB) led to mito- 
chondrial dysfunction and that CSA/CSB loss disrupts normal polymerase progression at G4 structures in neuroblastoma cells. They went on to show with a Caenorhabditis elegans model that ligand-induced G4 stabilization causes PARP1 activation and accelerated aging, suggesting that defective rDNA transcription of G4-laden DNA underlies a ramped-up DNA damage response and abnormal genome homeostasis. In a follow-up study, Okur et al. [2020] reported that CSA and CSB both interact with nucleolin, a major nucleolar protein that preferentially binds to G4 DNA and regulates rDNA transcription and ribosome biogenesis in a manner that is orchestrated with CSA/CSB.

Other hereditary disorders classified as ribosomopathies may have defects in G4 DNA metabolism [FarleyBarnes et al., 2019; Kampen et al., 2020]. These would include Diamond-Blackfan anemia, $5 \mathrm{q}$ syndrome, Shwachman-Diamond syndrome, X-linked dyskeratosis congenita, cartilage hair hyperplasia, and Treacher Collins syndrome. However, future studies are required to verify whether such conditions result from either polymerase I deficiency or abnormal G4 metabolism or both. Strikingly, most of these syndromes have an increased propensity to develop various cancers, a characteristic of many helicase disorders [Dhar et al., 2020].

\section{G4 DNA Stabilization Offers a Synthetic Lethal Approach to Attack Cancer}

The fundamental nature of cancer is that it relies upon the accumulation of mutations that result in the inactivation of tumor suppressor genes and activation of cancerpromoting proto-oncogenes. Understanding the molecular basis for structural variation in human cancer and how aberrations in processes like DNA replication lead to genomic rearrangements and mutagenesis and promote oncogenesis in specific cancer types has gained traction with advances in new sequencing technologies [ $\mathrm{Li}$ et al., 2020]. For example, the Balasubramanian lab developed a technique whereby genome-wide mapping of endogenous G4 was accomplished by a combined G4-specific antibody immunoprecipitation and high-throughput sequencing [Hänsel-Hertsch et al., 2018]. Interest in G4 landscapes characteristic of specific tumor types (e.g., breast cancer) may help to stratify anti-cancer treatment strategies in new ways, such as targeting G4 DNA with small molecules [Hänsel-Hertsch et al., 2020]. Although the landscape of G4 DNA structural regions in breast cancer patient-derived tumor xenograft models was deter- mined, a comparison to normal tissues/cells was not made, making it difficult to conclude the differences between the apparent G4 landscapes of normal versus cancerous cells. It is plausible that the number of G4 structures would be more abundant in cancer cells owing to increased DNA content (hyperdiploid or polyploidy), but this hypothesis remains to be tested.

Nucleoli which house rDNA/ribosomes are distinct regions within nuclei. In metastatic cancer cells the number and size of nucleoli are significantly elevated [Weeks et al., 2019], raising the possibility that G4 DNA landscape in the rDNA repeats may be differentially altered. Changes in nucleoli status may occur during different cell cycle phases which would likely have consequences for G4 DNA assembly. Using a G4-specific fluorescent probe that enables single-molecule and real-time detection of individual G4 structures in living cells, Di Antonio et al. [2020] showed that G-quadruplexes rise during S-phase of the cell cycle, suggesting their enrichment during DNA replication. The observation that in higher eukaryotes nucleoli usually disappear in mitosis and ribosome production commences at the end of mitosis with an increase in G1 followed by a maximal increase in G2 suggests that the locations where G4 accumulates in rDNA may be dynamic and influenced by cell cycle status. This is potentially important in considering the effect of G4 DNA on chromatin structure in nucleoli and, more generally, nuclei. For example, G4 DNA may exclude assembly of the histone octamer and wrapping of duplex DNA to form nucleosomes. Further studies in this area are required because there are likely to be consequences for transcriptional regulation and other genomic processes (e.g., DNA repair). The notion that transcription influences G4 status in vivo is supported by a recent observation that transcription is accompanied by a robust formation of G4 in active genes of living cells, as demonstrated using an artificial protein with high affinity and specificity for Gquadruplexes [Zheng KW et al., 2020].

Cancer cells, with their elevated requirements in metabolism, energy production, and biomolecule synthesis, are known to require an elevated level of rDNA transcription to ensure adequate cellular protein levels [Derenzini et al., 2000]. This has prompted cancer biologists to consider pharmacological inhibition of rDNA transcription as a potential avenue to preferentially reduce the proliferative capacity of cancer cells. Indeed, a number of small molecule compounds which inhibit rDNA transcription have been described which induce apoptosis of various cancer cell types [Drygin et al., 2009, 2011; Bywater et al., 2012]. Xu et al. [2017] examined the 
molecular and cellular mechanism whereby one such compound (CX-5461) in Phase I clinical trials for haematologic malignancies operates. CX-5461, like several other RNA pol I inhibitors, was found to bind and stabilize G4 DNA structures in vitro [Xu et al., 2017], which presumably interferes with expression of guanine-rich rDNA prone to form G4. However, there is more to the story. The researchers determined that CX-5461 preferentially kills cancer cells that are defective in the genome stability and DNA repair factors BRCA1 or BRCA2, which also play instrumental roles in protecting stalled or blocked replication forks. Colon cancer cells (HCT116, DLD1) defective in BRCA2 were also shown to be sensitive to CX-5461 [Xu et al., 2017]. Thus, ligand-induced G4 stabilization may represent an effective strategy for helping to eradicate BRCA1/2 deficient tumors. However, there are questions recently being raised over the mechanism whereby certain G4 ligands exert their biological activity against tumors. For example, experimental evidence was recently provided that the G4-binding compound pyridostatin promotes trapping of topoisomerase II on DNA by promoting G4 stabilization [Olivieri et al., 2020]. It was speculated that CX-5461 may operate by a similar mechanism. Irrespective of this, it seems probable that the ability of CX-5461 to exert its anticancer effects extends beyond its ability to inhibit RNA pol I transcription.

As nicely summarized in review articles from the laboratories of Seimiya [Nakanishi and Seimiya, 2020] and Johnson [Johnson, 2020], targeting G-quadruplexes in cancer cells with G4 ligands to inflict DNA damage and impair proliferation is a plausible approach to the development of novel chemotherapeutic strategies. Whether rDNA represents a viable target in amplifying the DNA damaging effects of G4 ligand-induced stabilization and fine-tuning the efficacy of such an approach remains to be determined. Ample experimental studies suggest that the ability to modulate expression of oncogenes richly characterized by predicted G4-forming sequences in their promoter elements or meddle with telomere maintenance mechanisms involving telomerase or the alternate lengthening of telomeres pathways offer novel and promising anti-cancer strategies. A distinct challenge will be to gain an improved understanding of how cancers with existing DNA repair deficiencies can be targeted with G4 ligands, sparing normal cells and tissues from the deleterious effects of G4 stabilization.

G4-resolving helicases are a prioritized class of proteins to target in synthetic lethality (SL) approaches to combat cancer [McLuckie et al., 2013; Datta et al., 2021].

Ribosomal DNA, G-Quadruplexes, and Disease
It is plausible that SL with G4-interacting ligands could be achieved by genetic SL or chemically induced SL with G4-interacting proteins such as DNA helicases and DNA repair factors as reasonable targets. Indeed, cancer cells with deficiency in the tumor suppressors BRCA1 or BRCA2 implicated in homologous recombination and stalled replication fork stabilization were found to be hypersensitive to the G4-stabilizing compound pyridostatin [Zimmer et al., 2016]. Our own work demonstrated that cancer cells deficient in the G4-resolving helicase FANCJ were highly sensitive to the G4 ligand telomestatin in terms of cell proliferation, apoptosis, DNA damage induction and depressed DNA replication [Wu et al., 2008]. A more recent study employing a genome-wide shRNA screen with A375 human melanoma cells identified genetic vulnerabilities to the G4 ligands pyridostatin and PhenDC3 in a diverse group of genes encoding DNA damage response factors or DNA helicases (RTEL1, RECQL4, DHX36) [Zyner et al., 2019]. Interestingly, "ribosome" is among one of the 5 significantly enriched gene pathway clusters for G4 sensitization. It should be kept in mind that G4 sensitization may be dependent on (1) compound dose, (2) genotype, and (3) cell line [Zyner et al., 2019]. In addition to a genetic deficiency pre-existing in a tumor or induced by RNA interference/gene targeting, G4-resolving helicases may be amenable to chemical inhibition by pharmacologically active small molecules [Hengel et al., 2017; Datta and Brosh, 2018]. Further studies in this area are warranted.

\section{Concluding Remarks}

In this review, we focused our discussion on the evidence that rDNA represents a difficult-to-replicate sequence that requires specialized proteins, such as DNA helicases, to resolve unusual DNA structures, including G4, formed by the repeat elements. Problems with rDNA stability, accumulation of damage, and defective replication and transcription contribute to certain hereditary disorders and age-related diseases by disrupting normal cellular homeostasis. While these themes are largely supported by studies of model organisms, clearly more work in mammalian systems and human cells is required, where our understanding is much more limited. Adding to the complexity, it is becoming increasingly apparent that diverse factors/mechanisms such as DNA base oxidation and base excision repair proteins [Roychoudhury et al., 2020], as well as chromatin state in which G4 formation may alter DNA wrapping into nucleosomes 
[Johnson, 2020], will demand more sophisticated analyses to fully appreciate how specific chromosomal loci are affected by G-quadruplexes. Future work may move the field of anti-cancer therapy forward with the further development of pharmacologically active G4-stabilizing ligands that target G4 at rDNA and other loci and induce DNA damage in a SL manner in tumors with existing DNA repair deficiencies.

\section{Acknowledgments}

We wish to thank the members of our respective laboratories at Bethany College and the National Institute on Aging, NIH for helpful discussions.

\section{Conflict of Interest Statement}

All authors have no conflicts of interest to declare.

\section{Funding Sources}

This work was supported by the Intramural Research Program of the National Institute on Aging, NIH.

\section{Author Contributions}

All authors contributed to the concepts presented in the manuscript and the writing of the manuscript.

\section{References}

Andrs M, Hasanova Z, Oravetzova A, Dobrovolna J, Janscak P. RECQ5: A Mysterious Helicase at the Interface of DNA Replication and Transcription. Genes (Basel). 2020;11(2):232.

Balajee AS, May A, Dianov GL, Friedberg EC, Bohr VA. Reduced RNA polymerase II transcription in intact and permeabilized Cockayne syndrome group B cells. Proc Natl Acad Sci USA. 1997;94(9):4306-11.

Ben-Shem A, Jenner L, Yusupova G, Yusupov M. Crystal structure of the eukaryotic ribosome. Science. 2010;330(6008):1203-9.

Bharti SK, Awate S, Banerjee T, Brosh RM. Getting Ready for the Dance: FANCJ Irons Out DNA Wrinkles. Genes (Basel). 2016;7(7):31.

Biffi G, Tannahill D, McCafferty J, Balasubramanian S. Quantitative visualization of DNA Gquadruplex structures in human cells. Nat Chem. 2013;5(3):182-6.

Bradsher J, Auriol J, Proietti de Santis L, Iben S, Vonesch JL, Grummt I, et al. CSB is a component of RNA pol I transcription. Mol Cell. 2002;10(4):819-29.

Brosh RM Jr, Cantor SB. Molecular and cellular functions of the FANCJ DNA helicase defective in cancer and in Fanconi anemia. Front Genet. 2014;5:372.

Budhathoki JB, Maleki P, Roy WA, Janscak P, Yodh JG, Balci H. A Comparative Study of GQuadruplex Unfolding and DNA Reeling Activities of Human RECQ5 Helicase. Biophys J. 2016;110(12):2585-96.

Bugaut A, Balasubramanian S. 5'-UTR RNA Gquadruplexes: translation regulation and targeting. Nucleic Acids Res. 2012;40(11):472741.

Burge S, Parkinson GN, Hazel P, Todd AK, Neidle S. Quadruplex DNA: sequence, topology and structure. Nucleic Acids Res. 2006;34(19): 5402-15.
Byrd AK, Zybailov BL, Maddukuri L, Gao J, Marecki JC, Jaiswal M, et al. Evidence That G-quadruplex DNA Accumulates in the Cytoplasm and Participates in Stress Granule Assembly in Response to Oxidative Stress. J Biol Chem. 2016;291(34):18041-57.

Bywater MJ, Poortinga G, Sanij E, Hein N, Peck A, Cullinane $\mathrm{C}$, et al. Inhibition of RNA polymerase I as a therapeutic strategy to promote cancer-specific activation of p53. Cancer Cell. 2012;22(1):51-65.

Chambers VS, Marsico G, Boutell JM, Di Antonio M, Smith GP, Balasubramanian S. Highthroughput sequencing of DNA G-quadruplex structures in the human genome. Nat Biotechnol. 2015;33(8):877-81.

Chatterjee S, Zagelbaum J, Savitsky P, Sturzenegger A, Huttner D, Janscak P, et al. Mechanistic insight into the interaction of BLM helicase with intra-strand G-quadruplex structures. Nat Commun. 2014;5:5556.

Chiarella S, De Cola A, Scaglione GL, Carletti E, Graziano V, Barcaroli D, et al. Nucleophosmin mutations alter its nucleolar localization by impairing G-quadruplex binding at ribosomal DNA. Nucleic Acids Res. 2013;41(5): 3228-39.

Christians FC, Hanawalt PC. Repair in ribosomal RNA genes is deficient in xeroderma pigmentosum group $\mathrm{C}$ and in Cockayne's syndrome cells. Mutat Res. 1994;323(4):179-87.

Datta A, Dhar S, Awate S, Brosh RM Jr. Synthetic Lethal Interactions of RECQ Helicases. Trends Cancer. 2021;7(2):146-161.

Datta A, Brosh RM Jr. New Insights Into DNA Helicases as Druggable Targets for Cancer Therapy. Front Mol Biosci. 2018;5:59.

Del Villar-Guerra R, Trent JO, Chaires JB. GQuadruplex Secondary Structure Obtained from Circular Dichroism Spectroscopy. Angew Chem Int Ed Engl. 2018;57(24):7171-5.
Derenzini M, Trerè D, Pession A, Govoni M, Sirri V, Chieco P. Nucleolar size indicates the rapidity of cell proliferation in cancer tissues. J Pathol. 2000;191(2):181-6.

Dhar S, Datta A, Brosh RM Jr. DNA helicases and their roles in cancer. DNA Repair (Amst). 2020;96:102994.

Dianov GL, Houle JF, Iyer N, Bohr VA, Friedberg EC. Reduced RNA polymerase II transcription in extracts of cockayne syndrome and xeroderma pigmentosum/Cockayne syndrome cells. Nucleic Acids Res. 1997;25(18):363642.

Di Antonio M, Ponjavic A, Radzevicius A, Ranasinghe RT, Catalano M, Zhang X, et al. Singlemolecule visualization of DNA G-quadruplex formation in live cells. Nat Chem. 2020;12(9): 832-7.

Dorn A, Feller L, Castri D, Röhrig S, Enderle J, Herrmann NJ, et al. An Arabidopsis FANCJ helicase homologue is required for DNA crosslink repair and rDNA repeat stability. PLoS Genet. 2019;15(5):e1008174.

Drosopoulos WC, Kosiyatrakul ST, Schildkraut CL. BLM helicase facilitates telomere replication during leading strand synthesis of telomeres. J Cell Biol. 2015;210(2):191-208.

Drygin D, Siddiqui-Jain A, O'Brien S, Schwaebe M, Lin A, Bliesath J, et al. Anticancer activity of CX-3543: a direct inhibitor of rRNA biogenesis. Cancer Res. 2009;69(19):7653-61.

Drygin D, Lin A, Bliesath J, Ho CB, O'Brien SE, Proffitt C, et al. Targeting RNA polymerase I with an oral small molecule CX-5461 inhibits ribosomal RNA synthesis and solid tumor growth. Cancer Res. 2011;71(4):1418-30.

Eddy J, Maizels N. Gene function correlates with potential for G4 DNA formation in the human genome. Nucleic Acids Res. 2006;34(14): 3887-96. 
Farley-Barnes KI, Ogawa LM, Baserga SJ. Ribosomopathies: Old Concepts, New Controversies. Trends Genet. 2019;35(10):754-67.

Fousteri M, Vermeulen W, van Zeeland AA, Mullenders LH. Cockayne syndrome A and B proteins differentially regulate recruitment of chromatin remodeling and repair factors to stalled RNA polymerase II in vivo. Mol Cell. 2006;23(4):471-82.

Gao H, Chen XB, McGowan CH. Mus81 endonuclease localizes to nucleoli and to regions of DNA damage in human S-phase cells. Mol Biol Cell. 2003;14(12):4826-34.

Goodfellow SJ, Zomerdijk JC. Basic mechanisms in RNA polymerase I transcription of the ribosomal RNA genes. Subcell Biochem. 2013; 61:211-36.

Gorgoulis V, Adams PD, Alimonti A, Bennett DC, Bischof O, Bishop C, et al. Cellular Senescence: Defining a Path Forward. Cell. 2019; 179(4):813-27.

Gray MD, Wang L, Youssoufian H, Martin GM, Oshima J. Werner helicase is localized to transcriptionally active nucleoli of cycling cells. Exp Cell Res. 1998;242(2):487-94.

Guo JU, Bartel DP. RNA G-quadruplexes are globally unfolded in eukaryotic cells and depleted in bacteria. Science. 2016;353(6306): aaf5371.

Hamadeh Z, Lansdorp P. RECQL5 at the Intersection of Replication and Transcription. Front Cell Dev Biol. 2020;8:324.

Hanakahi LA, Sun H, Maizels N. High affinity interactions of nucleolin with G-G-paired rDNA. J Biol Chem. 1999;274(22):15908-12.

Hänsel-Hertsch R, Spiegel J, Marsico G, Tannahill D, Balasubramanian S. Genome-wide mapping of endogenous G-quadruplex DNA structures by chromatin immunoprecipitation and high-throughput sequencing. Nat Protoc. 2018;13(3):551-64.

Hänsel-Hertsch R, Simeone A, Shea A, Hui WWI, Zyner KG, Marsico G, et al. Landscape of Gquadruplex DNA structural regions in breast cancer. Nat Genet. 2020;52(9):878-83.

Hardin CC, Watson T, Corregan M, Bailey C. Cation-dependent transition between the quadruplex and Watson-Crick hairpin forms of d(CGCG3GCG). Biochemistry. 1992; 31(3):833-41.

Hengel SR, Spies MA, Spies M. Small-Molecule Inhibitors Targeting DNA Repair and DNA Repair Deficiency in Research and Cancer Therapy. Cell Chem Biol. 2017;24(9):110119.

Henning KA, Li L, Iyer N, McDaniel LD, Reagan MS, Legerski $\mathrm{R}$, et al. The Cockayne syndrome group A gene encodes a WD repeat protein that interacts with CSB protein and a subunit of RNA polymerase II TFIIH. Cell. 1995;82(4):555-64

Hershman SG, Chen Q, Lee JY, Kozak ML, Yue P, Wang LS, et al. Genomic distribution and functional analyses of potential G-quadruplex-forming sequences in Saccharomyces cerevisiae. Nucleic Acids Res. 2008;36(1): 144-56.
Huppert JL, Balasubramanian S. Prevalence of quadruplexes in the human genome. Nucleic Acids Res. 2005;33(9):2908-16.

Ibrahim F, Maragkakis M, Alexiou P, Mourelatos Z. Ribothrypsis, a novel process of canonical mRNA decay, mediates ribosome-phased mRNA endonucleolysis. Nat Struct Mol Biol. 2018;25(4):302-10.

Ide S, Miyazaki T, Maki H, Kobayashi T. Abundance of ribosomal RNA gene copies maintains genome integrity. Science. 2010; 327(5966):693-6.

Indig FE, Rybanska I, Karmakar P, Devulapalli C, $\mathrm{Fu} \mathrm{H}$, Carrier F, et al. Nucleolin inhibits G4 oligonucleotide unwinding by Werner helicase. PLoS One. 2012;7(6):e35229.

Iyer LM, Leipe DD, Koonin EV, Aravind L. Evolutionary history and higher order classification of AAA+ ATPases. J Struct Biol. 2004; 146(1-2):11-31.

Joachimi A, Benz A, Hartig JS. A comparison of DNA and RNA quadruplex structures and stabilities. Bioorg Med Chem. 2009;17(19): 6811-5.

Johnson FB. Fundamentals of G-quadruplex biology. Annu Rep Med Chem. 2020;54:3-44.

Kamath-Loeb A, Loeb LA, Fry M. The Werner syndrome protein is distinguished from the Bloom syndrome protein by its capacity to tightly bind diverse DNA structures. PLoS One. 2012;7(1):e30189.

Kampen KR, Sulima SO, Vereecke S, De Keersmaecker K. Hallmarks of ribosomopathies. Nucleic Acids Res. 2020;48(3):1013-28.

Karikkineth AC, Scheibye-Knudsen M, Fivenson E, Croteau DL, Bohr VA. Cockayne syndrome: Clinical features, model systems and pathways. Ageing Res Rev. 2017;33:3-17.

Kief DR, Warner JR. Hierarchy of elements regulating synthesis of ribosomal proteins in Saccharomyces cerevisiae. Mol Cell Biol. 1981; 1(11):1016-23.

Kikin O, D'Antonio L, Bagga PS. QGRS Mapper: a web-based server for predicting G-quadruplexes in nucleotide sequences. Nucleic Acids Res. 2006;34(Web Server issue):W676-82.

Kobayashi T. A new role of the rDNA and nucleolus in the nucleus--rDNA instability maintains genome integrity. Bioessays. 2008;30(3): 267-72.

Koch S, Garcia Gonzalez O, Assfalg R, Schelling A, Schäfer P, Scharffetter-Kochanek K, et al. Cockayne syndrome protein A is a transcription factor of RNA polymerase I and stimulates ribosomal biogenesis and growth. Cell Cycle. 2014;13(13):2029-37.

Kormuth KA, Woolford JL Jr, Armitage BA. Homologous PNA Hybridization to Noncanonical DNA G-Quadruplexes. Biochemistry. 2016;55(12):1749-57.

Kwok CK, Marsico G, Sahakyan AB, Chambers VS, Balasubramanian S. rG4-seq reveals widespread formation of G-quadruplex structures in the human transcriptome. Nat Meth ods. 2016;13(10):841-4.
Lebedev A, Scharffetter-Kochanek K, Iben S. Truncated Cockayne syndrome B protein represses elongation by RNA polymerase I. J Mol Biol. 2008;382(2):266-74.

Li Y, Roberts ND, Wala JA, Shapira O, Schumacher SE, Kumar K, et al. Patterns of somatic structural variation in human cancer genomes. Nature. 2020;578(7793):112-21.

McLuckie KI, Di Antonio M, Zecchini H, Xian J, Caldas C, Krippendorff BF, et al. G-quadruplex DNA as a molecular target for induced synthetic lethality in cancer cells. J Am Chem Soc. 2013;135(26):9640-3.

McStay B, Grummt I. The epigenetics of rRNA genes: from molecular to chromosome biology. Annu Rev Cell Dev Biol. 2008;24:131-57.

Mendoza O, Bourdoncle A, Boulé JB, Brosh RM Jr, Mergny JL. G-quadruplexes and helicases. Nucleic Acids Res. 2016;44(5):1989-2006.

Mergny JL, Li J, Lacroix L, Amrane S, Chaires JB. Thermal difference spectra: a specific signature for nucleic acid structures. Nucleic Acids Res. 2005;33(16):e138.

Millevoi S, Moine H, Vagner S. G-quadruplexes in RNA biology. Wiley Interdiscip Rev RNA. 2012;3(4):495-507.

Mohaghegh P, Karow JK, Brosh RM Jr, Bohr VA, Hickson ID. The Bloom's and Werner's syndrome proteins are DNA structure-specific helicases. Nucleic Acids Res. 2001;29(13): 2843-9.

Muellner J, Schmidt KH. Yeast Genome Maintenance by the Multifunctional PIF1 DNA Helicase Family. Genes (Basel). 2020;11(2):224.

Mukundan VT, Phan AT. Bulges in G-quadruplexes: broadening the definition of G-quadruplex-forming sequences. J Am Chem Soc. 2013;135(13):5017-28

Nakanishi C, Seimiya H. G-quadruplex in cancer biology and drug discovery. Biochem Biophys Res Commun. 2020;531(1):45-50.

Okur MN, Lee JH, Osmani W, Kimura R, Demarest TG, Croteau DL, et al. Cockayne syndrome group $\mathrm{A}$ and $\mathrm{B}$ proteins function in rRNA transcription through nucleolin regulation. Nucleic Acids Res. 2020;48(5):247385.

Olivieri M, Cho T, Álvarez-Quilón A, Li K, Schellenberg MJ, Zimmermann M, et al. A Genetic Map of the Response to DNA Damage in $\mathrm{Hu}-$ man Cells. Cell. 2020;182(2):481-496.e21.

Paeschke K, Capra JA, Zakian VA. DNA replication through G-quadruplex motifs is promoted by the Saccharomyces cerevisiae Pif1 DNA helicase. Cell. 2011;145(5):678-91.

Palumbo SL, Ebbinghaus SW, Hurley LH. Formation of a unique end-to-end stacked pair of G-quadruplexes in the hTERT core promoter with implications for inhibition of telomerase by G-quadruplex-interactive ligands. J Am Chem Soc. 2009;131(31):10878-91.

Paramasivan S, Rujan I, Bolton PH. Circular dichroism of quadruplex DNAs: applications to structure, cation effects and ligand binding. Methods. 2007;43(4):324-31. 
Peculis BA. Ribosome biogenesis: ribosomal RNA synthesis as a package deal. Curr Biol. 2002;12(18):R623-4.

Petr MA, Tulika T, Carmona-Marin LM, Scheibye-Knudsen M. Protecting the Aging Genome. Trends Cell Biol. 2020;30(2):117-32.

Roychoudhury S, Pramanik S, Harris HL, Tarpley M, Sarkar A, Spagnol G, et al. Endogenous oxidized DNA bases and APE1 regulate the formation of G-quadruplex structures in the genome. Proc Natl Acad Sci USA. 2020; 117(21):11409-20.

Sabouri N. The functions of the multi-tasking Pfh1(Pif1) helicase. Curr Genet. 2017;63(4): 621-6.

Sabouri N, McDonald KR, Webb CJ, Cristea IM, Zakian VA. DNA replication through hardto-replicate sites, including both highly transcribed RNA Pol II and Pol III genes, requires the S. pombe Pfh1 helicase. Genes Dev. 2012; 26(6):581-93.

Sabouri N, Capra JA, Zakian VA. The essential Schizosaccharomyces pombe Pfh1 DNA helicase promotes fork movement past G-quadruplex motifs to prevent DNA damage. BMC Biol. 2014;12:101.

Sarker AH, Tsutakawa SE, Kostek S, Ng C, Shin DS, Peris M, et al. Recognition of RNA polymerase II and transcription bubbles by XPG, CSB, and TFIIH: insights for transcriptioncoupled repair and Cockayne Syndrome. Mol Cell. 2005;20(2):187-98.

Sauer M, Paeschke K. G-quadruplex unwinding helicases and their function in vivo. Biochem Soc Trans. 2017;45(5):1173-82.

Scheibye-Knudsen M, Tseng A, Borch Jensen M, Scheibye-Alsing K, Fang EF, Iyama T, et al. Cockayne syndrome group A and B proteins converge on transcription-linked resolution of non-B DNA. Proc Natl Acad Sci USA. 2016;113(44):12502-7.

Selby CP, Sancar A. Cockayne syndrome group B protein enhances elongation by RNA polymerase II. Proc Natl Acad Sci USA. 1997; 94(21):11205-9.

Shiratori M, Suzuki T, Itoh C, Goto M, Furuichi Y, Matsumoto T. WRN helicase accelerates the transcription of ribosomal RNA as a component of an RNA polymerase I-associated complex. Oncogene. 2002;21(16):2447-54.
Sinclair DA, Guarente L. Extrachromosomal rDNA circles--a cause of aging in yeast. Cell. 1997;91(7):1033-42.

Sinclair DA, Mills K, Guarente L. Accelerated aging and nucleolar fragmentation in yeast sgs 1 mutants. Science. 1997;277(5330):1313-6.

Song J, Perreault JP, Topisirovic I, Richard S. RNA G-quadruplexes and their potential regulatory roles in translation. Translation (Austin). 2016;4(2):e1244031.

Srivastava R, Srivastava R, Ahn SH. The Epigenetic Pathways to Ribosomal DNA Silencing. Microbiol Mol Biol Rev. 2016;80(3):545-63.

Storci G, Bacalini MG, Bonifazi F, Garagnani P, De Carolis S, Salvioli S, et al. Ribosomal DNA instability: An evolutionary conserved fuel for inflammaging. Ageing Res Rev. 2020;58: 101018.

Sun H, Yabuki A, Maizels N. A human nuclease specific for G4 DNA. Proc Natl Acad Sci USA. 2001;98(22):12444-9.

Tantin D. RNA polymerase II elongation complexes containing the Cockayne syndrome group B protein interact with a molecular complex containing the transcription factor IIH components xeroderma pigmentosum B and p62. J Biol Chem. 1998;273(43):27794-9.

Tantin D, Kansal A, Carey M. Recruitment of the putative transcription-repair coupling factor CSB/ERCC6 to RNA polymerase II elongation complexes. Mol Cell Biol. 1997;17(12): 6803-14.

Urban V, Dobrovolna J, Hühn D, Fryzelkova J, Bartek J, Janscak P. RECQ5 helicase promotes resolution of conflicts between replication and transcription in human cells. J Cell Biol. 2016;214(4):401-15.

van Gool AJ, Citterio E, Rademakers S, van Os R, Vermeulen W, Constantinou A, et al. The Cockayne syndrome B protein, involved in transcription-coupled DNA repair, resides in an RNA polymerase II-containing complex. EMBO J. 1997;16(19):5955-65.

Vannier JB, Sarek G, Boulton SJ. RTEL1: functions of a disease-associated helicase. Trends Cell Biol. 2014;24(7):416-25.

Wallgren M, Mohammad JB, Yan KP, Pourbozorgi-Langroudi P, Ebrahimi M, Sabouri N. G-rich telomeric and ribosomal DNA sequences from the fission yeast genome form stable G-quadruplex DNA structures in vitro and are unwound by the Pfh1 DNA helicase. Nucleic Acids Res. 2016;44(13):6213-31.
Warmerdam DO, Wolthuis RMF. Keeping ribosomal DNA intact: a repeating challenge. Chromosome Res. 2019;27(1-2):57-72.

Weeks SE, Metge BJ, Samant RS. The nucleolus: a central response hub for the stressors that drive cancer progression. Cell Mol Life Sci. 2019;76(22):4511-24.

Wu Y, Shin-ya K, Brosh RM Jr. FANCJ helicase defective in Fanconia anemia and breast cancer unwinds G-quadruplex DNA to defend genomic stability. Mol Cell Biol. 2008;28(12): 4116-28.

Xu H, Di Antonio M, McKinney S, Mathew V, Ho B, O'Neil NJ, et al. CX-5461 is a DNA G-quadruplex stabilizer with selective lethality in BRCA1/2 deficient tumours. Nat Commun. 2017;8:14432.

Yu Y, Pham N, Xia B, Papusha A, Wang G, Yan $Z$, et al. Dna2 nuclease deficiency results in large and complex DNA insertions at chromosomal breaks. Nature. 2018;564(7735): 287-90.

Yuan X, Feng W, Imhof A, Grummt I, Zhou Y. Activation of RNA polymerase I transcription by cockayne syndrome group B protein and histone methyltransferase G9a. Mol Cell. 2007;27(4):585-95.

Zhang JY, Zheng KW, Xiao S, Hao YH, Tan Z. Mechanism and manipulation of DNA:RNA hybrid G-quadruplex formation in transcription of G-rich DNA. J Am Chem Soc. 2014; 136(4):1381-90.

Zheng KW, Zhang JY, He YD, Gong JY, Wen CJ, Chen JN, et al. Detection of genomic G-quadruplexes in living cells using a small artificial protein. Nucleic Acids Res. 2020;48(20): 11706-20.

Zheng L, Meng Y, Campbell JL, Shen B. Multiple roles of DNA2 nuclease/helicase in DNA metabolism, genome stability and human diseases. Nucleic Acids Res. 2020;48(1):16-35.

Zimmer J, Tacconi EMC, Folio C, Badie S, Porru $\mathrm{M}$, Klare $\mathrm{K}$, et al. Targeting BRCA1 and BRCA2 Deficiencies with G-Quadruplex-Interacting Compounds. Mol Cell. 2016;61(3): $449-60$.

Zyner KG, Mulhearn DS, Adhikari S, Martinez Cuesta S, Di Antonio M, Erard N, et al. Genetic interactions of G-quadruplexes in humans. Elife. 2019;8:e46793. 